originally absorbed be great or small." $\mathrm{He}$ evidently forgets that this remark applies only to the specific infectious, and not to the specific non-infectious, fevers, which differ from the former in many important particulars.

Moreover, in any of the specific infectious fevers, there is quite as marked a distinction between mild and severe cases, as there is hetween Ague and Remittent Fever, and yet no one has ever suggested that they are not one and the same disease. Upholders of the germtheory explain the differences by saying that the amount of the special pabulum of the disease differs in different cases: why should this not apply equally to the diseases under consideration?

(2) Dr. O'Connell draws attention to the fact that, whereas hypertrophy of the spleen, leucocythæmia, and pigmentary deposits are the common sequelæ of Ague, they are not so of Remittent Fever; and, therefore, he does not think that the two diseases can be due to the same morbid cause. Surely, to the most superficial observer it must be manifest that these changes are the result of long continued malarial poisoning, and that, therefore, it would be ridiculous to expect that a single attack of Remittent Fever (or indeed Ague) of from five to twenty days' duration could produce them.

His theory as to the causation of Intermittent Fever by malarial climate is as follows:-

(1) The two essentials are heat and moisture, which, by preventing its excretion, lead to an intermittent excess of water in the blood.

(2) This intermittent excess of water in the blood $(a)$ increases tissue metamorphosis, and (b), when it reaches a certain point, arrests evaporation, thus producing fever. If the increase of water be intermittent, so will be the fever.

This theory is very ingeniously argued out, and tables are given contrasting the meteoroJogical conditions at Ambala in April and August 1882, and giving the daily admissions for Ague during those months. From a perusal of these it appears that, coincidently with an increase in the relative humidity of the air and a decrease in the daily range of temperature, there was an increase in the number of admissions for ague. The number of observations, however, are too few for any sound theory to be based upon them. The great fault in Dr. O'Conuell's work is, that he has quite overlooked the distinction between primary and secondary attacks of Ague. It is well known that a change in the hygrometric state of the atmosphere, which would have no appreciable effect upon a man, who had never been exposed to malarial influences, is quite sufficient to produce a paroxysm of Ague in one who has undergone this exposure. Unless, therefore, it can be clearly demonstrated that primary attacks of Ague are due to meteorological and climatic changes, his theory falls to the ground. As a matter of fact, a glance at the table of admissions for August almost convinces us that his cases must have been secondary ones, for, when we note that the highest daily number of admissions was 4 (and that only on two days in the month), and then take into consideration the fact that the whole of the troops were exposed to the same atmospheric conditions, it appears highly improbable that these can have constituted the most important factor in the causation of the disease. It is more reasonable to assume either that these cases occurred in men previously saturated with the malarial poison, and consequently more susceptible to atmospheric influences, or that the heat and moisture generated fresh malarial poison in places where the local conditions were favourable to its development.

Diagnosis and Surgical Treatment of Abdominal Tumours. By Sir Spencer Wells, BART. Londion: J. \& A. Churchill, 1885.

THIs is a fourth edition of Sir. Spencer Wells' well-known work, the first edition of which was published in 1865. The subjectmatter has been somewhat condensed, and much new material added. It is got up in the style of Sir Henry 'Thompson's cheap edition of his work on the bladder. The greater portion of the book is devoted to the consideration of ovarian and allied tumours. It is a thoroughly practical work, well written and illustrated, and is a valuable record of the author's long experience and work, which in great part form the history of ovariotomy. The chapter on the rise and progress of this operation is both interesting and instructive. As regards the influence of Listerism in lessening the mortality after ovariotomy, the author expresses himself somewhat guardedly. He says: "Much may be due to antiseptic dressings, and to the persistent diffusion of an antiseptic atmosphere: but it must be remembered that, at the same time, my patients, since 1878, have all had not only the advantages belonging to a position in life superior to that of hospital cases, but they have been exempt from the risks of the possible importation of hospital contagion. The abandonment of the clamp, and the use of the ligature with the intra-peritoneal treatment of the pedicle took place at the same time as the introduction of the antiseptic treatment."

It is a matter of regret that, in the chapter on Oophorectomy, the valuable and original work of Mr. Lawson Tait is completely ignnred. We should not have expected so serious an omission from a man of Sir Spencer Wells' reputation. 
The remainder of the work deals with the operative treatment of tumours other than ovarian or uterine, such as splenic, renal, hepatic, \&c. This part of the subject is only treated in a very general way, so much so indeed as scarcely to justify the adoption of such a title as "Diagnosis and Surgical 'Treatment of Abdominal Tumours." For instance, the operations of lumbar nephrotomy and nephrectomy are not even mentioned. It is in fact little more than a record of the cases of abdominal tumour operated upon by himself.

There is one point on which we must differ from the author. When discussing the propriety of extirpation of the spleen in certain cases, he says that the best results are to be hoped for in cases of simple or malarial hypertrophy. Indian experience, however, goes to prove that extirpation of the spleen is quite inadmissible in the latter disease.

\section{Ģbstricts and cettracts.}

\section{USE OF ANTIPYRINE IN SUNSTROKE.}

\section{By Benjamin F. Westbrook, M.D.,}

\section{Physician-in-Chief to the Department of Diseases of the} Chest, St. Mary's General Hospital, Brooklyn.

Mr object in publishing this note is to call attention to the use of antipyrine in two cases of sunstroke, so that others may, if they think proper, test it, and contribute the results of their experience.

It occurred to me that the prompt introduction of an antipyretic dose of this substance into the subcutaneous tissues might be of great service in reducing the temperature, and so give time for attention to the treatment of the other pathological conditions. The ambulance surgeon, Dr. Folker, was therefore requested to provide himself with a fifty-per-cent. solution, and, in the event of his being called to a case of the apoplectic form of the disease, to administer a drachm of the solution hypodermically at once, and to repeat it in an hour, if the temperature was not markedly reduced. Other measures for the relief of the patient were, of course, not to be neglected.

On Saturday, the 18th, when the thermometer registered $99^{\circ} \mathrm{F}$. in the shade, he was called to attend a young Englishman, twenty-two years old, of very robust habit, who, after working out of doors all the forenoon, became comatose and convulsed after reaching his home at midday. The head was congested, eyes suffused, pulse rapid and strong, skin dry, and the coma profound. The bowels had moved spontaneously, and he had vomited freely. The rectal temperature was $109^{\circ} \mathrm{F}$. The doctor administered a drachm of the fifty-per-cent. solution of antipyrine hypodermically, applied ice to the head, and removed him to the hospital.

In the confusion attending his admission the exact time was not noted, but in about three-quarters of an hour after the first dose the rectal temperature was $107 \cdot 50^{\circ} \mathrm{F}$. Cold was applied to the body and head by means of towels wrung out of ice-water, and frequently changed; and another drachm of the solution was introduced under the skin. In thirty minutes the rectal temperature was again taken, and found to be $99^{\circ} \mathrm{F}$. The cold applications were at once discontinued, and dry heat was applied to the surface, while whisky was injected subcutaneously.

When I arrived shortly afterward the surface of the body was cool, the pulse 120, the coma profound, and all the limbs were twitching convulsively. The head was still congested, the conjunctivæ injected, the pupils contracted but responding feebly to light, and the respiration was irregular and accompanied by a loud expiratory groan, such as is heard in severe cerebral disturbance. I gave two ounces of whisky and forty grains of chloral hydrate by enema, but they were not retained. About half an ounce of whisky was then given hypodermically. In half an hour the temperature began to rise slowly, so that the surface was warm. The twitching of the voluntary muscles became more marked, and he was seized with a most violent tetanic convulsion, which was controlled by chloroform. As the convulsions succeeded each other with increasing rapidity and intensity, I opened the median basilic vein, and withdrew thirteen ounces of blood. The bleeding was terminated sooner than was desirable by the onset of a terrific convulsion, during which the arm was twisted violently and the opening in the vein occluded. After this there were no more severe convulsions; the patient became more quiet, and the rectum retained forty grains of chloral hydrate. This was followed by a period of rest, during which the axillary temperature was taken and found to be $103 \cdot 75^{\circ} \mathrm{F}$.

One drachm of Lente's solution was thrown under the skin, and repeated about once in two hours till four doses were administered. At 8-15 P.M., as slight convulsions had occurred from time to time, six leeches were applied to the temporal regions, twenty grains of antipyrine given hypodermically, and forty grains of chloral hydrate by the rectum. From this time on he slept quietly, the temperature slowly declining until Sunday morning, when it was $99^{\circ} \mathrm{F}$.

At about ten o'clock on Saturday evening the patient first became sufficiently conscious to partially respond when spoken to, and from this he passed into a condition of hebetude, which, by Monday forenoon, had given place to complete consciousness. The temperature has been normal since Sunday afternoon, though the pulse is still somewhat rapid. He takes nourishment well. From Sunday morning to Monday morning he had forty grains of sodium bromide every two hours, and five grains of the sulphate of quinine every four hours. The doses were then reduced to twenty grains and two grains.

This case represented the severest type of sunstroke. I have never before seen so bad a one recover. The temperature was high, the coma very profound, and the convulsions the most terrible that $I$ have ever witnessed, except in one case of tetanus, the subject of which died within twenty-four hours after the diagnosis was made. The rapid and permanent reduction of temperature, which must have been mainly due to the antipyrine-inasmuch as the cold applications were only continued for half an hour-undoubtedly contributed toward the favourable result by giving time and removing at once the injurious effect of the byperpyretic 